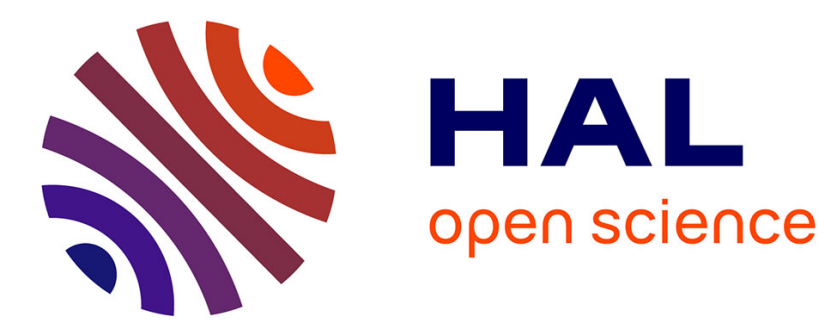

\title{
Generation of polycrystalline material at the atomic scale
}

B Mantisi

\section{To cite this version:}

B Mantisi. Generation of polycrystalline material at the atomic scale. Computational Materials Science, 2016, 118, pp.245-250. 10.1016/j.commatsci.2016.03.002 . hal-01310400

\section{HAL Id: hal-01310400 https://hal.sorbonne-universite.fr/hal-01310400}

Submitted on 2 May 2016

HAL is a multi-disciplinary open access archive for the deposit and dissemination of scientific research documents, whether they are published or not. The documents may come from teaching and research institutions in France or abroad, or from public or private research centers.
L'archive ouverte pluridisciplinaire HAL, est destinée au dépôt et à la diffusion de documents scientifiques de niveau recherche, publiés ou non, émanant des établissements d'enseignement et de recherche français ou étrangers, des laboratoires publics ou privés. 


\title{
Generation of polycrystalline material at the atomic scale
}

\author{
B. Mantisi \\ Laboratoire de Physique Théorique de la Matière Condensée, Paris Sorbonne Universités UPMC, Boite 121, 4 place \\ Jussieu 75252 Paris Cedex 05 France
}

\begin{abstract}
Polycrystalline structure plays an important role in the macroscopic properties of a solid material. In this paper we propose a new code to generate a polycrystalline material at the atomic scale. Our polycrystalline systems are based on the random generation from initial germs. From a mechanical point of view, it brings new features as for example ductility of 3D polycrystalline aluminum. By using molecular dynamics simulations, we also show the effect of temperature on a geological material, olivine, one of the most abundant silicate mineral of the Earth upper mantle. The importance of porous media is also considered (e.g. metals).
\end{abstract}

\section{Introduction}

The importance of atomic dislocations on the mechanical behaviour of solid materials is well known and the study of polycrystalline materials is important in that respect. Polycrystalline materials are common in nature. For example, in metallurgy, grain boundaries will generate new macroscopic behaviours compare with a mono-crystalline phase. In geophysics, the polycrystallinity of rocks is a rule. Moreover the Earth mantle being submitted to extreme conditions of temperature and pressure, the influence of the grain boundaries on mechanical and transport properties of rocky mantle is then important to know. A fruitful way to approach the pressure and temperature dependence of these materials is to use molecular dynamics (MD) simulations.

MD simulations are widely used in many fields as mechanics, physics, geology and biology. The main ingredient is the force field (FF), which governs the interaction between particles. The validity of the simulation results depends directly on the accuracy of the FF. Furthermore, for polycrystalline materials the initial configuration of the atoms in the simulation cell has to be as realistic as possible. In general, molecular dynamics codes of the literature give the possibility to build only mono-crystalline structures [1]. Some other codes were developed to build more complex crystalline structures made of proteins [2] or polymers [3]. But, concerning polycrystalline material, users face up to a difficult task. The challenge is to built a polycrystalline structure including a part of randomness. An obvious way would be to proceed as in a real experiment: i.e. cooling a liquid slow enough to let appear and grow mono crystalline germs. This method works well for metals simulated by MD [4] for which single symmetries (fcc or bcc) facilitate the growth from a small nucleus. However, the success of the method is potential dependent and does not work necessarily for every kind of materials (e.g. silicates). Another method consists in building crystalline cells with a Voronoi tessellation [5] [6], and assigns to each crystalline area a random orientation. The main advantages of this method is that it is independent of the FF, it is scalable to different sizes and applicable to different kind of simulations (MD and finite element methods for example). However, all grains are separated by planes defined a priori, whereas some roughness is expected at grain boundaries. Moreover, no porosity can be introduced in that way to model polycrystalline structures. Moreover, atomistic simulation of polycrystalline materials has shown already nice results [7] and therefore a code especially devoted to build such initial structure would be a good starting point. In this context, we propose to build a polycrystalline material from the growth of mono-crystals which is stopped when such crystals join each other within the simulation box. The code produces at the atomic scale a random configuration representative of a polycrystalline system. Here, randomness means a random distribution of initial germs with random orientation and random growth. Generated polycrystals are an as- 
sembly of several perfect monocrystals, i.e. defects are not introduced inside a grain. In section II we present the basic ideas of the method and how the algorithm works. In section III some details of the code are given. In section IV a first example presents the effect of polycrystallinity on the plasticity of aluminum. The second example deals with the partial melting of olivine, a silicate mineral of the Earth upper mantle. We conclude and give some perspectives in section $\mathrm{V}$.

\section{Description of the code}

The code uses a geometrical criterion based on the unit cell structure (UCS) of the material under investigation. Figure 1 sketches the process discussed below. The algorithm follows a growth process, which implies to consider two kinds of chemical entities (An entity is the unit cell of the considered crystal or a subunit of this unit cell). Chemical entities that do exist (RE for real entities) and chemical entities that can potentially exist (VE for virtual entities) as first neighbours of REs. The initial state is a distribution of REs (germs) to which we assign a random location and a random orientation in the simulation box. These germs are the first RE in the box. A virtual grid mapped on the crystal symmetry is associated with each germ. The second step is the crystal growth of these initial germs from their associated grid. For that, VE are defined as nearest neighbour of RE on their grid, and then algorithm pick up randomly a VE and declares it as RE. The new RE implies first to delete VEs (i.e VEs from other germs) closer than the minimum distance between entities defined from the UCS and second to declare new VE following the grid of the last defined RE. The process is stopped when the grids are filled taking into account that entities of different grids can not overlap each others. An important task of the code is to deal with the configurations where entities belonging to different grains become close to each other. The growth process is very efficient since all random pick up are accepted, because VE have been defined such that they do not overlap with any other RE. To avoid boundary effects during the simulation, the code considers periodic boundary conditions when writing RE and VE.

Some accelerating features, discussed in the next part, have been implemented, giving then access to large system sizes in an acceptable generating time. The main accelerating feature is a subdivision of

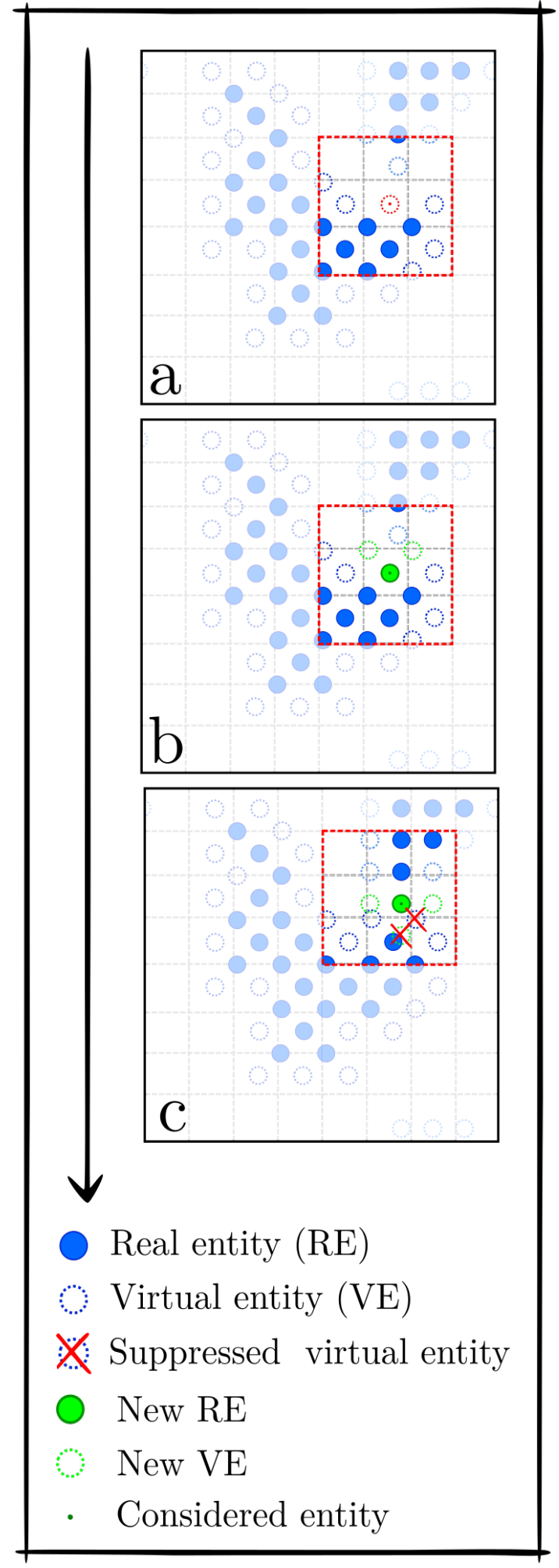

Figure 1: [Color Online] a. One VE is selected, b. and declared as RE. New VE are then defined. c. Another VE is selected and declared as RE. If it overlap with existing $\mathrm{VE}$, the latter are suppressed. If New VE overlap with existing RE they are not created.

the box in sub boxes which decrease the number 
of computations. It is depicted in Figure 1 by the red square surrounding a set of sub boxes. Instead of computing all distances and checking a possible overlap with all atoms in the box, the computation deals with a small number of surrounded boxes (more precisely defined in the following). We plot in figure 2 the time it takes to generate system sizes reported on $\mathrm{x}$-axis for 3 -dimension and 2-dimension systems respectively. We note that for a 2 dimensional system, it takes less than one minute on a Intel Xeon E5-2650 @ $2.6 \mathrm{GHz}$ with gfortran compiler and just one core, to generate a configuration composed of 200000 atoms.
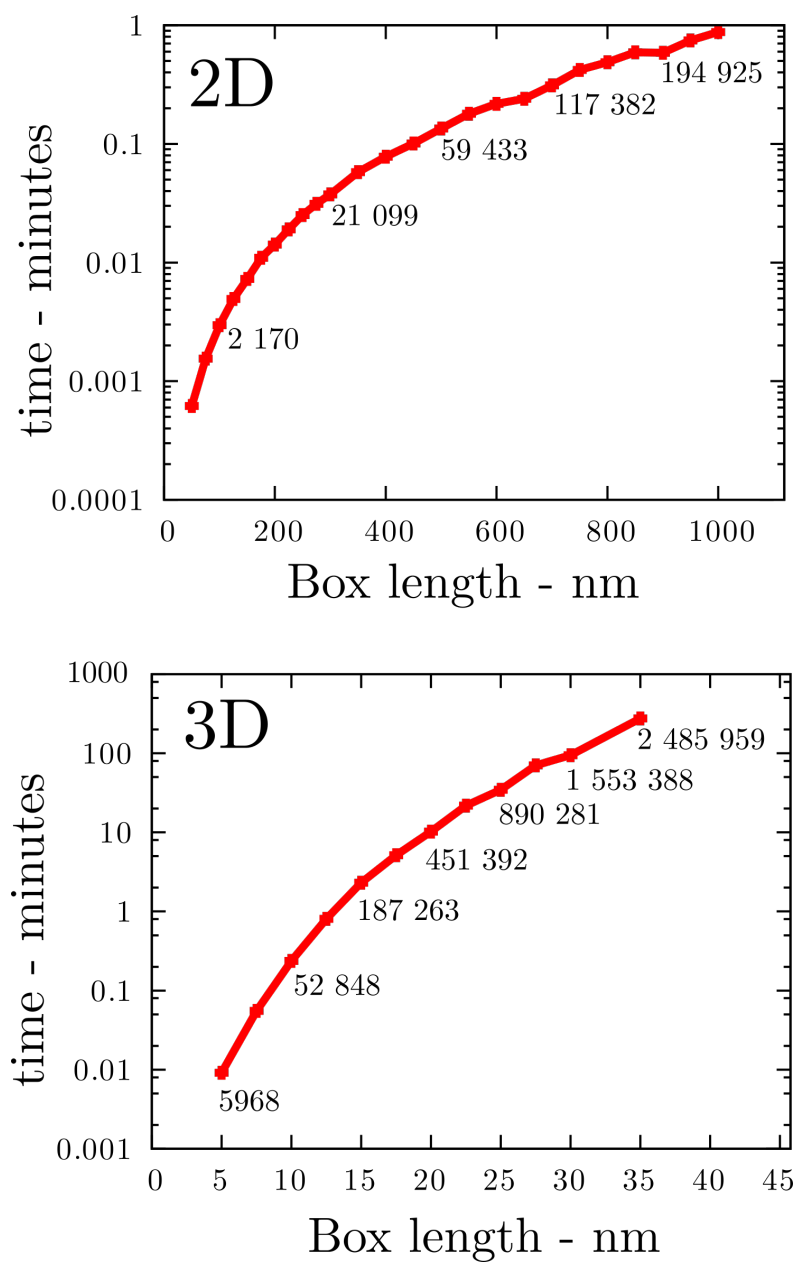

Figure 2: [Color Online] Time efficiency to generate polycrystalline systems. a. is for 3 dimensional systems and b. is for 2 dimensions. Labels on pictures refer to the number of atoms created. All computations were made on a Intel Xeon E5-2650 @ 2.6GHz core with gfortran compiler.

\section{Details on the code}

Grain growth. Figure 3 presents the two dimensional growth of 20 mono atomic germs with a cfc geometry in a 200x200 box units ( 1 box unit = atomic diameter). The separation between grain is imposed to be 2 box units. Since the algorithm is not a real growth process because temperature and then diffusion of species are not considered. Concerning the grains statistics evolution, the bigger the grains, the higher the number of $\mathrm{VE}$ around have to be considered, i.e more chance they have to be larger than others. By contrast, some mono-crystals are trapped, because they are closely surrounded by other mono-crystals precluding to them any further growth. The density of germs and the space between them when the simulation starts are thus very important.

Code optimisation. The code has been written to generate many different configurations of several hundred of thousand of atoms. Any loop on all atoms at each building step would be a lost of time. We reduce the computational time using sub boxes paving the simulation cell with sizes of a few angstroms. Then all atoms or entities are labelled in a sub box and the check for non-overlapping is done using nearest neighbour boxes. This is the main feature which renders the code so efficient (A two dimensional box containing around 200000 atoms is generated in less than one minute). The size of small boxes is related to the nearest neighbour distances within the unit cell of the crystal under investigation. This method applies to any crystal structure.

Adjustable parameters. The user can easily change the dimension of the box ( 2 or 3 dimensions), the size of the box, the number of grains, and the space between grains. The space between grains is equal or greater than the typical distance between two atomic species of the crystal unit cell. Whatever is the space imposed between the grains (porosity), the final configuration needs to be minimized in energy with an adequate FF. The code does not provide a minimization method, since existing molecular dynamics codes do it already pretty well. In the forthcoming, we will use LAMMPS for minimizing boxgenerated structures. During the minimization process, the free surfaces minimize their area, porosity is restructured (Figure 5). As we will see later, porosity at the nano-scale may 
bring new features in the behaviour of materials under extreme conditions.

Crystal orientation. The germ orientation is chosen at random when the simulation starts. In $3 \mathrm{D}$, for each germ we associate a unit vector built from two random points in a box, which is a rotation axis to which we associate a random rotation angle. In $2 \mathrm{D}, \mathrm{z}$-axis perpendicular to the system plane is the rotation axis, the rotation angle is chosen randomly. The user can specify the $\mathrm{x}-\mathrm{y}$ axis correspondence with the lattice parameters, and then the corresponding lattice rotation axis . For example, for $\vec{a}, \vec{b}$ and $\vec{c}$ lattice vector, one can specify that $\vec{a}$ is along the $\mathrm{z}$ axis, and $\vec{b}, \vec{c}$ along $\mathrm{x}, \mathrm{y}$ axis respectively. A cyclic permutation is done here. Note that in $2 \mathrm{~d}$ systems, the lattice vector along $\mathrm{z}$ will be the thickness of the sample. $L_{x}, L_{y}$ and $L_{z}$ box edges are specified, then the code will take as $L_{z}$ the integer times the lattice vector closer than the requested values(For instance, if $|\vec{c}|$ along $\mathrm{z}$ is $4 \AA$, and the requested value is $L_{z}=19 \AA$, then the final value will be $L_{z}=5 \cdot|\vec{c}|=20 \AA$ ).

Periodic boundary conditions. We choose to build the system with periodic boundary conditions (PBC). If one atom goes out from the box, then it position is shifted by the box size in the opposite direction. Thus, the system can be seen as an infinite continuum of grains periodically replicated. However, because of PBC, a grain may growth, cross the boundary and met itself. Since the initial orientation of the grain is random and not correlated with the size of the box, the probability to have a perfect match when both parts met each others is close to zero. To avoid this it is recommended to start at least with 6-8 grains for 2D simulations and 10-12 grains for 3D simulations.

Stoichiometry conservation. In order to keep the stoichiometry of the total sample constant, one needs to first characterize entities in the Unit Cell and be aware of possible atomistic local structures (e.g. $\mathrm{SiO}_{4}$ tetrahedron). For example the chemical composition of Olivine is $\mathrm{Mg}_{2}-\mathrm{SiO}_{4}$, the unit cell contains 28 atoms ( $4 \mathrm{Si}, 16 \mathrm{O}$ and $8 \mathrm{Mg}$ ) (figure 4a). We split the unit cell in four parts, each containing the correct stoichiometry of $\mathrm{Mg}_{2}-\mathrm{SiO}_{4}$. To do this and keep local geometries entities $\left(\mathrm{SiO}_{4}\right.$ tetrahedra) intact, we move oxygen atoms out of the box considering others unit cells around (arrows on figure 4a). The scheme of the code is in considering the center of mass of the defined sub unit cell structure to i) fill the box, and to ii) verify that there is no atomic overlap between the selected VE and surrounding REs. For mono-atomic structures such as fcc aluminum for example, there is no need to care of this.
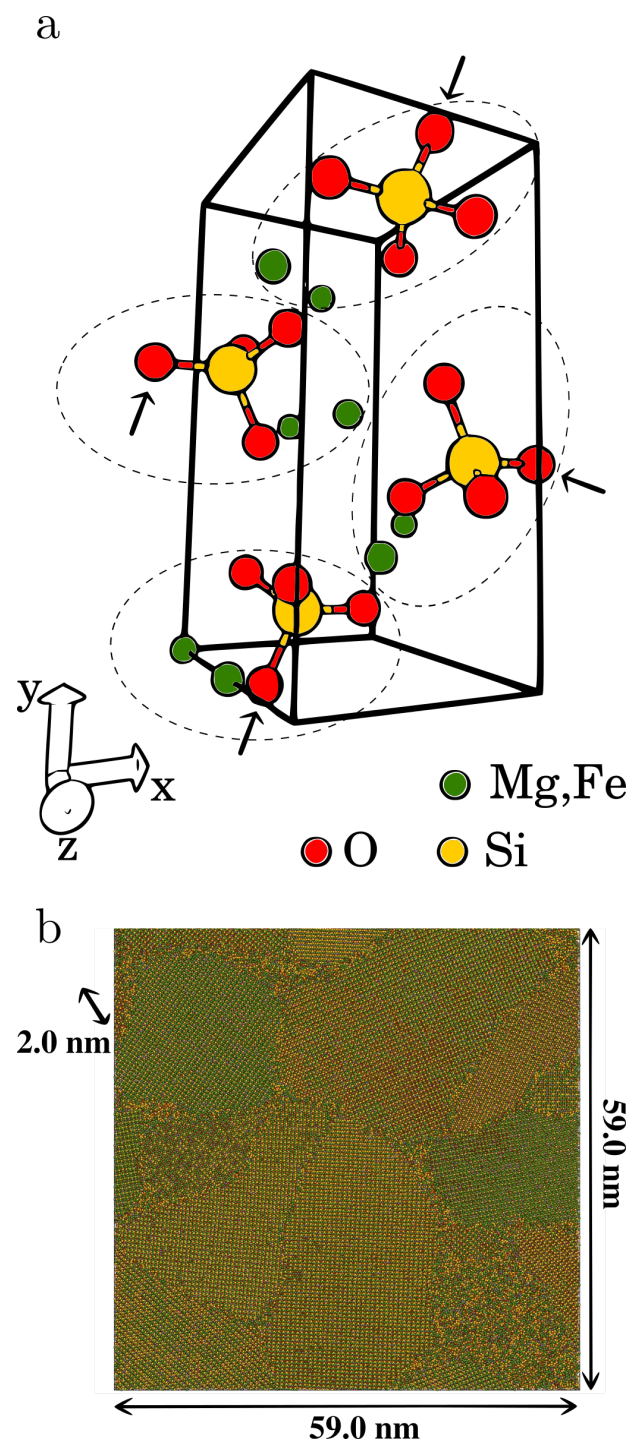

Figure 4: [Color Online] a. Olivine unit cell. Atoms grouped together with a dashed line are entities used to build crystal, which preserve neutrality and stoichiometry. Arrows point out atoms which are outside of the unit cell. b. Sample of Olivine, San Carlos type, at $1700 \mathrm{~K}$ and $1 \mathrm{GPa}$, which sizes $59 \times 59 \times 2 \mathrm{~nm}$.

This code was primarily designed to study multi component materials constitutive of the Earth mantle (e.g. silicate minerals). But, it can also help for the study of metallic alloys that are mechanically loaded, therefore serving for the study of mixed alloys and their diffusion of atomic species or as a 
starting point for kinetic Monte-Carlo simulations devoted to grain growth evolution.

\section{Examples}

\section{method of calculation}

For molecular dynamics simulations and energy minimization we used the LAMMPS code [1].

The centrosymmetric coefficient $(\mathrm{Cs})$ is used to characterize the local order and highlights grain boundaries. This coefficient is computed for each atoms $\mathrm{i}$ over its $\mathrm{N}$ first neighbours $\mathrm{j}$ as follows:

$$
C s_{i}=\left|\sum_{j}^{N}\left(\vec{r}_{i}-\vec{r}_{j}\right)\right|
$$

and is equal to zero if the atoms around are perfectly ordered. The number of neighbours $N$ is dependent of the crystal geometry we want to characterize (e.g. for fcc $N=12$ ).

The mechanical behaviour is studied by stress-strain curves: the strain is imposed and the stress is computed from the Irving-Kirkwood formula [8]:

$$
\underline{\underline{\sigma}}=\frac{1}{V} \sum_{i}^{N} \sum_{j \neq i}^{N}\left(m_{i} \vec{v}_{i} \cdot \vec{v}_{i}+\vec{r}_{i j} \cdot \vec{F}_{i j}\right)
$$

where $\mathrm{i}$ and $\mathrm{j}$ are labelled particles, $\mathrm{V}$ the volume of the sample, $\mathrm{N}$ the total number of particles, $\vec{v}_{i}$ the particule's i velocity, $\vec{r}_{i j}$ the distance vector between particle $\mathrm{i}$ and $\mathrm{j}$, and $\vec{F}_{i j}$ the force between particles $i$ and $j$ deriving from the associated interaction potential. Note, that this tensor is defined as symmetric since in the first part (kinetic) the velocities can commute, and concerning the second part the two vectors are collinear. This feature is in fact resulting from the action-reaction law and then applicable to multi-body interactions [9].

\section{Results}

We first present the example of an aluminum sample under mechanical load, and next the one of a polycrystalline olivine one submitted to high temperature and pressure. Because the FF used for aluminum is at very short range, our computational resources allow us to reach much bigger sample sizes than for silicate minerals where Coulombic interaction are present.
Example 1 : Stress-strain curve: from fragility to ductility. We build a 3 dimensional aluminum polycrystal in a cubic box (19.3x19.3x19.3nm) containing 403076 atoms. Figure 5 shows the simulation box after energy

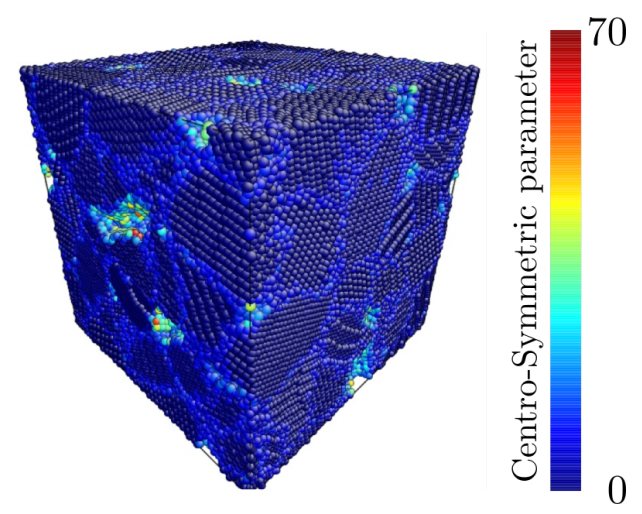

Figure 5: [Color Online] The box contains 403076 aluminum atoms and sizes $19.3 \mathrm{~nm}$ edge. 80 grains are generated and the space between grains is non zero. After energy minimization we clearly obtain voids which are found between grains. The color code represent the centrosymmetric parameter. This picture has been generated using AtomEye[10].

minimization. The FF used here is computed from the Embedded Atom Method (EAM) [11] [12] for Aluminum and tabulated by Mishin \& al [13]. The values are interpolated using spline function. Grain boundaries and free surfaces are characterised by $C s_{i} \neq 0$. The deformation was performed using minimization algorithm to avoid any shock wave due to the high strain rate (which is usual in classical MD due to the time scale); it gives access to the mechanical energy and does not take into account any thermal activation. Then the system is extended along the $\mathrm{x}$ direction in keeping constant the $y, z$ section of the box. The strain is applied by imposing a small displacement to atoms with a deformation step of $\epsilon=\Delta x / L_{0}=10^{-4}$. It is followed by a minimization of the local energy using a conjugated gradient method.

We compare the respective behaviour of a monocrystalline aluminum (lattice vector aligned along the box edge), a non-porous polycrystalline aluminum and a $1 \%$ porous polycrystalline aluminum. The stress-strain curve is represented in figure 6 by plotting the calculated Von Mises equivalent stress as function of the applied strain. The mono-crystalline sample is fragile but can sustain a high stress before breaking, here at $\epsilon=0.19$. 


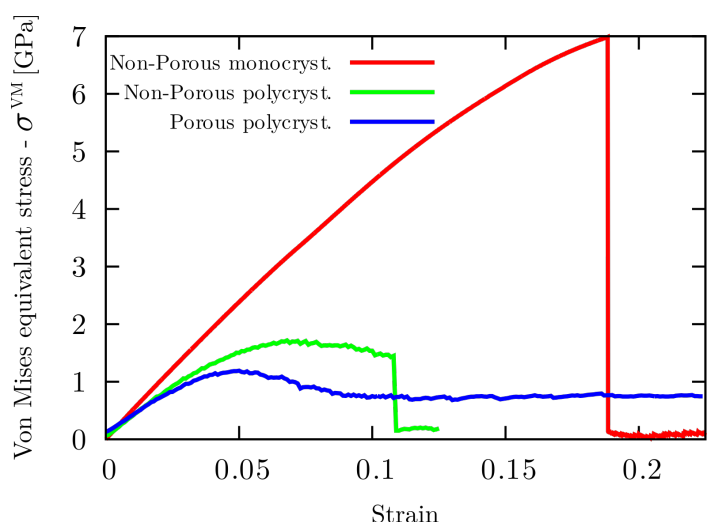

Figure 6: [Color Online] The stress-strain curves are plotted for an extension deformation of alluminium samples: a crystal of $16 \mathrm{~nm}$ size (red), a polycrystalline with 80 grains of $20 \mathrm{~nm}$ (green), a polycrystalline with 80 grains and voids of $20 \mathrm{~nm}$ (blue).

Concerning the polycrystalline system, one clearly notices a change from a fragile behaviour to a ductile one, and, indeed, the material flows under strain leading a much lower value of the yield stress. It is also well known that porosity plays a crucial role in the mechanical behaviour. Void growth is a topic largely developed in analytical mechanics (e.g. Gurson model [14]), and has recently been observed from molecular dynamics studies [15] [16] [17] [18]. Voids bring a new degree of freedom to redistribute energy. In fact, pores imply free surfaces which can store energy. In absence of free surfaces, the energy accumulates in the bulk and is released suddenly, creating cracks at the nanoscale. Figure 6 confirms this general tendency because the stress-strain curve of the porous material presents a larger ductility than the non-porous samples. Using the polycrystalline generation code, we thus clearly show the importance of the granular aspect of matter at the nanoscale on the macroscopic mechanical behaviour.

Example 2: Shear strain and grain boundary correlation. We build a pseudo 2 dimensional aluminum sample with sizes $79.7 \times 79.7 \times 2.0$ $\mathrm{nm}$ devoted to a shear strain along the $\mathrm{x}$-axis. The result of the grain growth code is presented on figure 7 with a color code associated to the local stress ( $\mathrm{xx}$ component of the stress tensor) for different shear strains. This color code clearly shows the grain boundaries. Figure 7 a presents a configuration which has been minimized using a conjugate gradient method. We use, as previously the EAM FF for describing the interactions between
$\mathrm{Al}$ atoms. Following figures $7 \mathrm{~b}, \mathrm{c}, \mathrm{d}$ and e represent the system for different shear strains. Several responses to the strain are observed here. First, defect appear and move but generally collapse in a grain boundary. Second, some cells tilt out from their original xy plane and this is one elementary mechanism for releasing stress. Third, grains on the upper side of the sample divide into smaller grains as long as the deformation occurs. This feature is quite unexpected and results from the displacement of aligned oscillating stress values similar to a dislocation. The orientation of crystals is not initially defined here, and one can observe the dislocation propagation and their interaction with other defects or even grain boundaries in the most general case. Moreover, when the sample is followed in time, one can note several dynamical features. Grain boundaries move, i.e. atoms belonging to a crystal and close to another grain can switch their orientation to be align along the neighbouring grain. We also clearly notice dislocation-like motion inside a grain. This example can be studied in more details by computing the associated Burger vector [20], characterizing grains size evolution during deformation or defect displacements.

\section{Example 3: Silicate minerals of Earth's} mantle : Polycrystalline olivine. Here we investigate the temperature effect on grain boundaries and diffusion located in channels between grains generated by the code. Channels in polycrystalline rocks are of considerable interest for the geophysical community, as they tend to dominate the electrical conductivity [22], and can be seen as an additional degrees of freedom.

Olivine is one of the most abundant constituent of the Earth's upper mantle. It is a silicate, whose unit cell composition is $\mathrm{Mg}_{2 x} \mathrm{Fe}_{2(1-x)} \mathrm{SiO}_{4}$. The presence of $\mathrm{Mg}$ and $\mathrm{Fe}$ cations leads to a solidus and a liquidus boundary in the phase diagram, where solid and liquid co-exist. Evaluation of the electrical conductivity has been done experimentally [23] [24] and unexpectedly the electrical conductivity, coming from the ionic transport, is high for a solid below the solidus. Molecular simulations can clearly bring a new insight in this field, in particular if the grain distribution can be fairly reproduced. This can be achieved with our code.

We build a pseudo 2 dimensional polycrystalline olivine in a cubic box of 59x59 nm edge, $2.0 \mathrm{~nm}$ thick 
and containing around 679000 atoms (Figure 4b). We use the accurate FF of Dufils et al. (2016) for describing the interaction in silicates. Once the polycrystal is generated, the potential energy is minimized using a conjugate gradient method. Next, the sample is equilibrated by MD at $300 \mathrm{~K}$ during $0.5 \mathrm{~ns}, 800 \mathrm{~K}$ during $0.5 \mathrm{~ns}, 1500 \mathrm{~K}$ during $3.0 \mathrm{~ns}$ and finally at $1700 \mathrm{~K}$ during $3.0 \mathrm{~ns}$. The pressure is fixed at $1 \mathrm{GPa}$. We compute the total displacement of the atoms at $1700 \mathrm{~K}$ during $3 \mathrm{~ns}$. The histogram of displacement is presented in figure 8a. Two types of displacement can be identify, one corresponding to the atomic motion within grains, and the other one associated with the melt (here melt is used for grain boundaries). The dichotomy is clearly visible in Figure 8a with a threshold at $1.7 \AA$, a distance which is similar to the Si-O bond length (1.6 $\AA$ ). Figure $8 \mathrm{~b}$ represents the simulation cell and highlights atoms which displace more than the threshold distance $(1.7 \AA)$. One notices that Atomic displacement are generally very small except at grain boundaries where a true diffusion can take place along grains. This finding supports phenomenological models attempting to estimate the electrical conductivity of polycrystals from basic geometries (planes, tubes, spheres...). But this is not in the scope of the present study.

\section{Conclusion}

In this article we have presented a code aimed to generate polycrystalline materials of various chemical composition and unit cell structure. This code generates the growth in 2 or 3D of a polycrystal from an initial distribution of randomly oriented crystalline germs. Moreover, it allows to control either space between grains or the porosity of the system (i.e. unit defaults or bigger voids).

Two model systems are investigated for illustration, a sample of polycrystalline aluminum sheared and extended and a silicate mineral (olivine) at high temperature. In most of the MD simulation studies of the literature only mono-crystals are considered although it is well known that polycrystallinity and grain boundaries deeply affect the mechanical and thermodynamic properties [7] [21]. An accurate description of solid materials accounting for polycrystallinity seems to be crucial to link microscopic behaviour to macroscopic ones. We are confident that the code presented here may be fruitful to build complex material at the nanoscale.
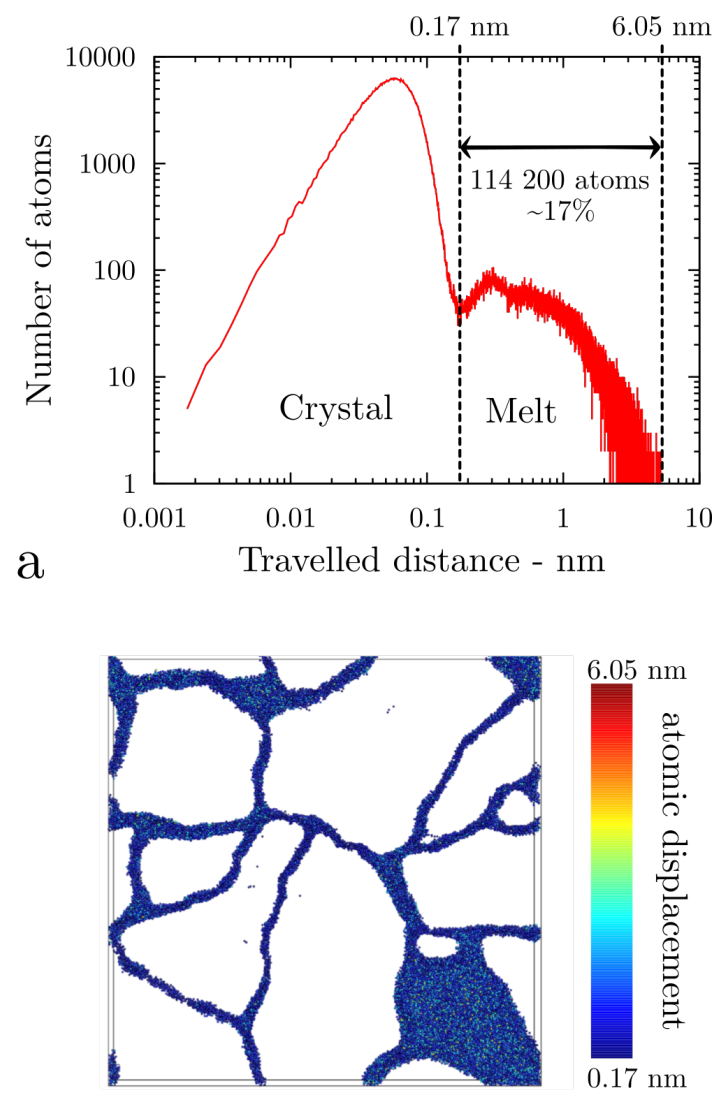

$\mathrm{b}$

Figure 8: [Color Online] a. histogram of displacements of each atoms after $3 \mathrm{~ns}$ at $1700 \mathrm{~K}$ and $1 \mathrm{GPa}$. b. Atoms which displaced more than $0.17 \mathrm{~nm}$. The region in white are grains.

\section{Acknowledgements}

The author gratefully acknowledge B. Guillot, N. Sator and M. Micoulaut for fruitful discussions and revision of the manuscript. This work was done with the financial support of the ERC grant $n^{\circ} 279790$.

\section{References}

[1] S. Plimpton, "Fast Parallel Algorithms for ShortRange Molecular Dynamics", J. Comp. Phys. 117, 1-19 (1995).

[2] L. Martinez, R. Andrade, E. G. Birgin, J. M. Martinez. Packmol: A package for building initial configurations for molecular dynamics simulations. Journal of Computational Chemistry, 30(13):21572164, 2009.

[3] V. Mainge, V. Jain, P.V. Bharatal and P.K. Maiti, "Dendrimer building toolkit: Model building and characterization of various dendrimer architecture", Journal of Computational Chemistry textbf33, 1997, (2012). 
[4] Y. Shibuta and T. Suzuki, "A molecular dynamics study of cooling rate during solidification of metal nanoparticles", Chemical physics Letters 502, 82 (2011).

[5] G. Voronoi, Z. Reine Angew. Math., 134 (1908), p. 199

[6] D. Chen, Comput. Mater. Sci., 3 (1995), p. 327

[7] J. Schiøtz "Atomic-scale modeling of plastic deformation of nanocrystalline copper", Scripta Mater. 51, 837 (2004)

[8] J. H. Irving and J. G. Kirkwood "The statistical mechanical theory of transport processes. IV. The equation of hydrodynamics" J. Chem. Phys. 18, 817 (1950).

[9] N. C. Admal and E. B. Tadmor "A unified interpretation of stress in molecular systems" J. Elast 100, 63-143 (2010).

[10] J. Li, Modelling Simul. Mater. Sci. Eng. 11, 173 (2003).

[11] M.S. Daw, M.I. Baskes "Semiempirical, quantum mechanical calculation of hydrogen embrittlement in metals" Physical Review Letters 50, 1285 (1983).

[12] M.S. Daw, M.I. Baskes "Embedded-atom method: Derivation and application to impurities, surfaces and other defects in metal" Physical Review B 29, 6443 (1984).

[13] Y. Mishin, D. Farkas, M.J. Mehl and D.A. Papaconstantopoulos "Interatomic potentials for monoatomic metals from experimental data and ab initio calculations" Physical review B 59, 3393 (1999).

[14] A.L. Gurson "Continuum theory of ductile rupture by void nucleation and growth: Part I, yield criteria and flow rules for porous ductile media" Journal of Engineering Materials and Technology 99, 2 (1977).

[15] G.P. Potirniche, M.F. Horstemeyer, G.J. Wagner, P.M. Gullett, "A molecular dynamics study of void growth and coalescence in single crystal nickel", International Journal of Plasticity 22, 257 (2006).

[16] C. Mi, D.A. Buttry, P. Sharma, D.A. Kouris, "Atomistic insights into dislocation-based mechanisms of void growth and coalescence" Journal of the Mechanics and Physics of Solids 59, 1858 (2011).

[17] S. Traiviratana, E.M. Bringa, D.J. Benson, M.A. Meyers "Void growth in metals: Atomistic Calculations" Acta Materialia 56, 3874 (2008).

[18] E.T. Seppala, J. Belak, and R.E. Rudd "Onset of Void Coalescence during Dynamic Fracture of Ductile Metals", Physical Review Letter 93, 245503 (2004).

[19] P. Keblinski, S.R. Phillpot, D. Wolf, H. Gleiter, Acta Mater., 45 (1997), p. 987
[20] A. Stukowski, K. Albe, Modelling Simul. Mater. Sci. Eng. 18025016 (2010).

[21] V. Yamakov, D. Wolf, S. R. Phillpot, A. K. Mukherjee and H. Gleiter "Dislocation processes in the deformation of nanocrystalline aluminium by molecular-dynamics simulation" Nature Materials 1, 45 (2002)

[22] T. Yoshino, T. Katsura "Electrical conductivity of mantle minerals: role of water in conductivity anomalies" Annu. Rev. Earth Planet. Sci. 41, 605 (2013)

[23] A. Pommier et. al. "Experimental investigation of the electrical behavior of olivine during partial melting under pressure and application to the lunar mantle" Earth and Planetary Science Letters 425, 242-255 (2015)

[24] K. Baba, A. D. Chave, R. L. Evans, G. Hirth, and R. L. Mackie, "Mantle dynamics beneath the East Pacific Rise at $17^{\circ}$ : Insights from the Mantle Electromagnetic and Tomography (MELT) experiment" J. Geophys. Res. 111, B02101 (2006)

[25] H. S. Waff, "Theoretical considerations of electrical conductivity in a partially molten mantle and implications for geothermometry, J. Geophys. Res., 79(26), 4003 (1974) 

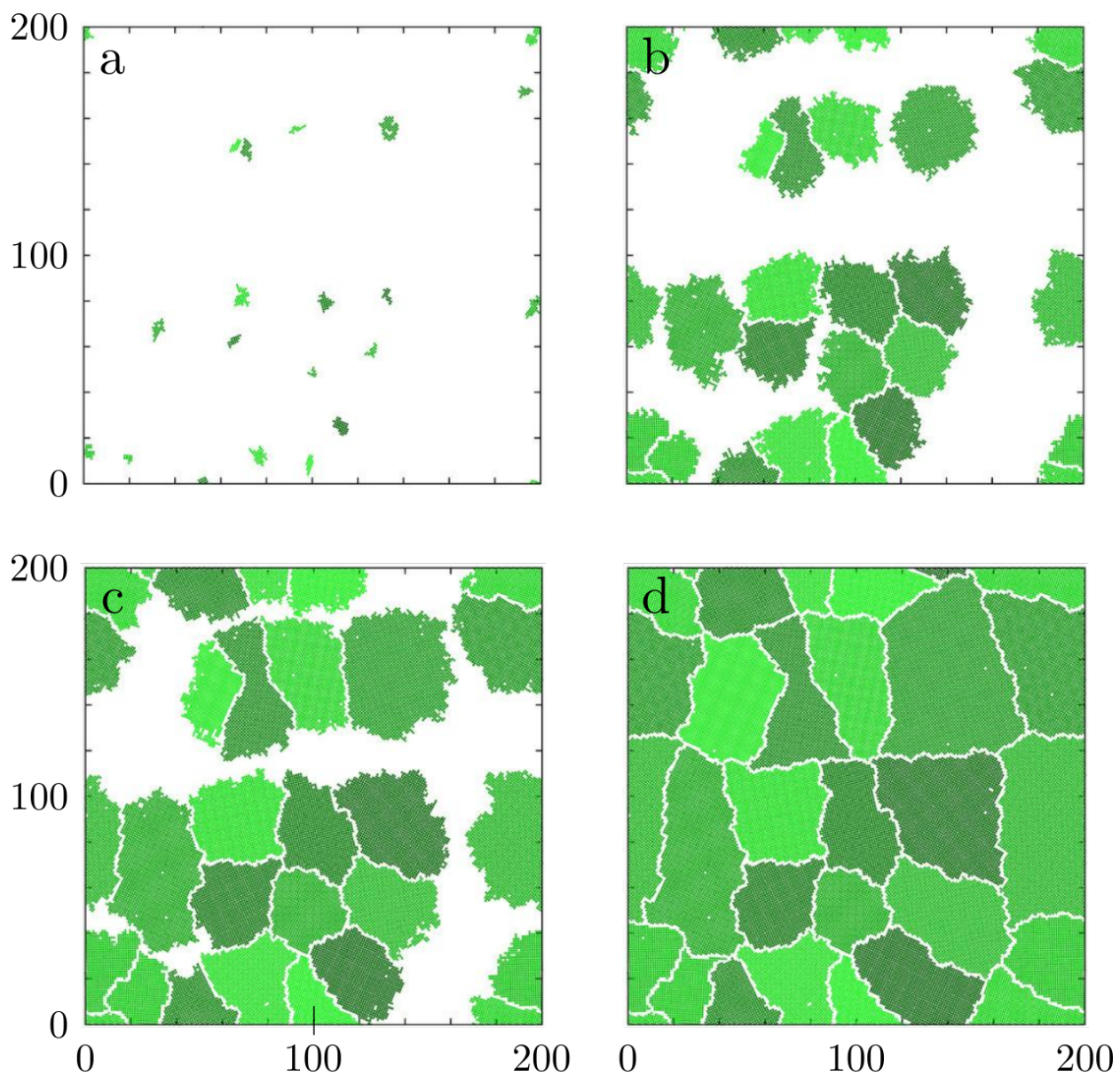

Figure 3: [Color Online] Growth of a model polycrystalline material in a plane. The unit cell is a cubic network. Colours are randomly affected for each grains. Separation between grains is an initial specification of at least 2 units here. 

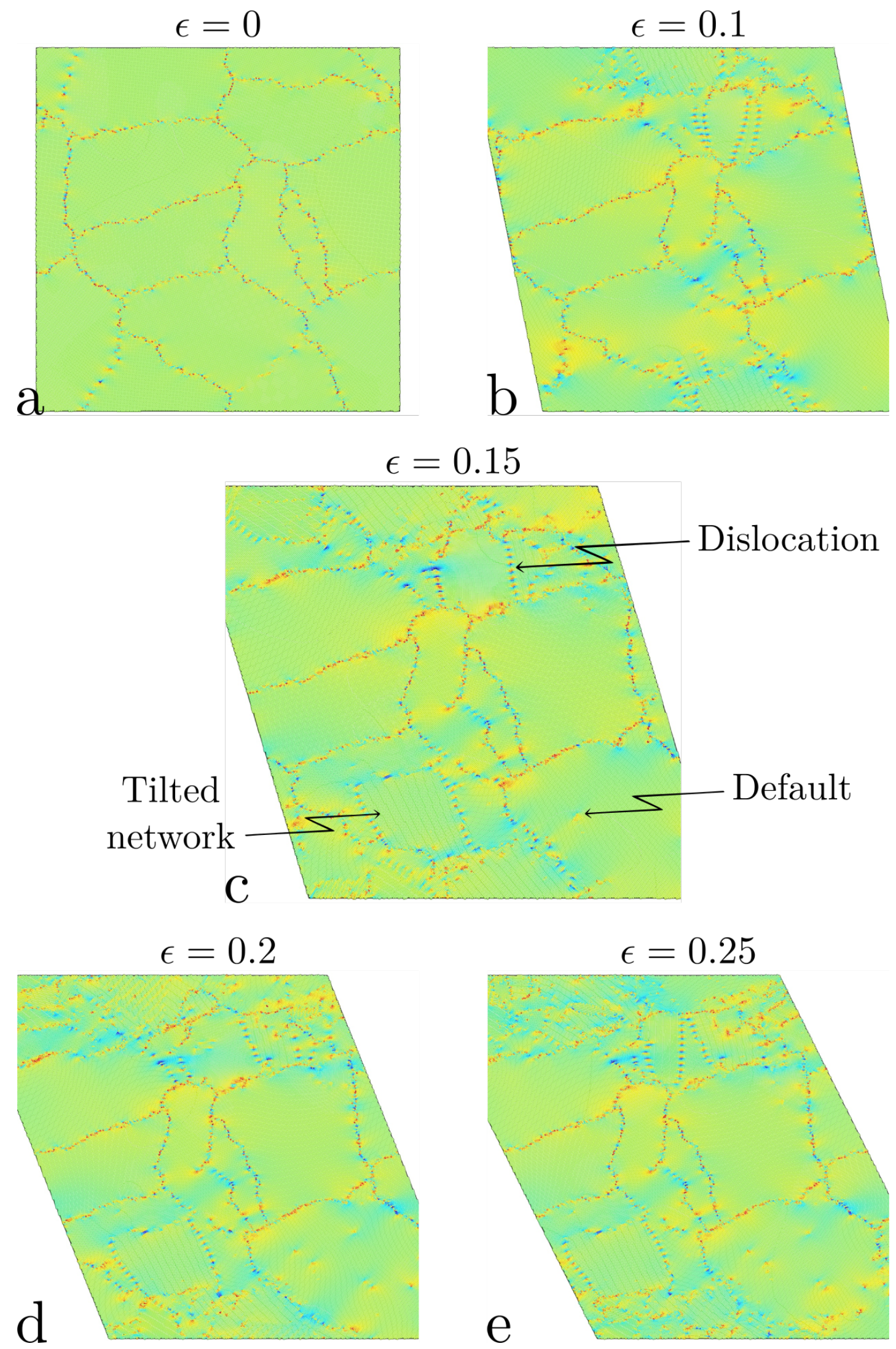

Figure 7: $\quad$ Color Online]Aluminum polycrystalline sample which sizes $79.7 \times 79.7 \times 2.0 \mathrm{~nm}$. Each picture represents different shear loads. The color map is related to the xx stress tensor component for each atom. It emphasizes defaults, dislocations and grain boundaries. The two firsts pictures are for 0 and 0.05 strain. The middle picture is at 0.15 strain and labels on it point out defaults, dislocations and tilted network. Finally pictures on bottom are from left to right 0.2 and 0.25 strain. 